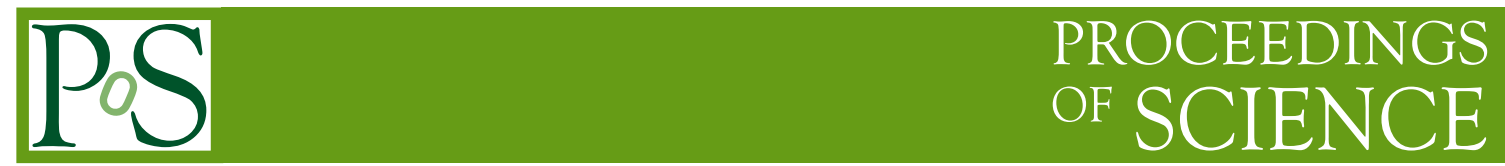

\title{
Hadron interactions
}

\author{
Kostas Orginos ${ }^{* \dagger}$ \\ College of William and Mary / JLab \\ E-mail: kostas@wm. edu
}

In this talk I am reviewing recent calculations of properties of multi-hadron systems in lattice QCD. In particular, I am reviewing results of elastic scattering phase shifts in meson-meson, meson-baryon and baryon-baryon systems, as well as discussing results indicating possible existence of bound states in two baryon systems. Finally, calculations of properties of systems with more than two hadrons are presented.

XXIX International Symposium on Lattice Field Theory

July $10-162011$

Squaw Valley, Lake Tahoe, California

* Speaker.

${ }^{\dagger}$ A footnote may follow. 


\section{Introduction}

From the very early times of lattice Quantum Chromodynamics (QCD), the importance of calculating properties of interacting hadrons had been recognized. However, the difficulty associated with such calculations on Euclidean space was also quickly recognized and solutions were proposed [1, 2, 3, 4]. Pioneering work focusing on toy problems started also quite early [5] and since then significant work has been done in calculating elastic scattering phase shifts, investigating the existence of bound states and resonances, as well as studying the properties of multi-hadron systems.

The interactions of hadrons originate from the underlying theory of strong interactions resulting complex emergent phenomena at low energies. Nuclear physics is a direct consequence of QCD and in principle all properties of atomic nuclei can be calculated from QCD. One of the major motivations of studying the interactions of hadrons in lattice QCD is precisely that: Understand how nuclear physics emerges from QCD, as well as compute from first principles some fundamental properties of nuclear matter. However, the large separation the characteristic scale of QCD $(\sim 1 \mathrm{GeV})$ and the low nuclear scales of a few $\mathrm{MeV}$ or even $\mathrm{KeV}$ makes such calculations a daunting task. For this reason only recently lattice QCD calculations directly targeting Nuclear Physics have started to be undertaken. In addition to Nuclear physics, hadronic interactions of two or more hadrons are essential in extracting information about resonances. In Euclidean space, as it was shown in [6], the spectrum of stable particles in a finite box in the channel where a resonance exists, can be used to extract the width and the energy of a resonance. Furthermore, calculations of Weak matrix elements of non-leptonic Weak decays of hadrons require [7, 8] the understanding of the finite volume spectrum of the final state multi-hadron system.

In this talk I am reviewing recent results from lattice QCD calculations of properties of interacting hadrons. Emphasis is given to results relevant to Nuclear physics.

\section{Hadron Interactions in Euclidean Space}

Computing the properties of an interacting hadronic system in Euclidean space requires a special treatment. In principle, one should calculate in Euclidean space appropriate correlations functions which after rotation to Minkowski they correspond to the desired scattering amplitude. However, analytic continuation cannot be simply performed using the Euclidean correlation functions on a discrete set of points. For this reason, it is desirable to extract the required information directly from Euclidean correlation functions. The simplest example where this is achieved is the calculation of the the spectrum of energy levels in a finite box, by observing the exponential decay of Euclidean correlation functions. Significant work has been performed nearly from the beginning of Lattice QCD formulation on understanding how properties of interacting hadrons can be calculated directly from Euclidean correlation functions. Pioneering work can be found in [1, 2, 3, 4, 6]. The basic principle behind this work is the fact that all the information about the interactions of hadrons is encoded in the energy spectrum of the the hadronic system in a box. Hence a calculation of this spectrum from Euclidean correlation functions can result in the calculation of the desired scattering amplitude. However, this is only possible where the analytic connection of the finite box spectrum to the desired scattering amplitude can be made. 


\subsection{Scattering and Bound States}

The simplest example where the idea described above can be applied is the calculation of the elastic s-wave scattering phase shift of two hadrons. The formalism described here was developed in $[2,4]$. In this case the scattering amplitude is described by a single phase (the scattering phase shift) $\delta(k)$.

$$
\mathscr{A}(k)=\frac{4 \pi}{\mu} \frac{1}{k \cot \delta(k)-i k}
$$

where $k$ is the magnitude of the center of mass scattering momentum and $\mu$ is the reduced mass of the two body system. The relation of this phase to the energy levels in a finite box can be easily worked out resulting for the case of equal mass particles of mass $\mu$.

$$
E_{n}=2 \sqrt{k_{n}^{2}+\mu^{2}}
$$

with $E_{n}$ being the energy spectrum of the two body system in a finite box, and $k_{n}$ the corresponding scattering momentum and,

$$
k \cot \delta(k)=\frac{1}{\pi L} S\left(\frac{k L}{2 \pi}\right), \quad S(\eta)=\lim _{\Lambda \rightarrow \infty}\left[\sum_{\vec{n}}^{|\vec{n}|<\Lambda} \frac{1}{|\vec{n}|^{2}-\eta^{2}}-4 \pi \Lambda\right]
$$

where $\vec{n}$ is a 3-vector with integer components. Thus in Euclidean space one calculates the energy levels $E_{n}$ using normal spectroscopic methods and extracts the scattering phase shift using the above formula. At small enough scattering momentum, where the effective range expansion is valid, the scattering length a can be identified through

$$
k \cot \delta(k)=\frac{1}{\mathrm{a}}+\frac{1}{2} \mathrm{r}^{2} k^{2}+\cdots
$$

with $r$ being the effective range of the interaction. Expanding at small $k$ one obtains the shift $\Delta E_{0}$ of the lowest two particle energy level in a box of size $L$ from the non-interacting two particle lowest energy level in the same box:

$$
\Delta E_{0}=-\frac{4 \pi \mathrm{a}}{m L^{3}}\left[1+c_{1} \frac{\mathrm{a}}{L}+c_{1} \frac{\mathrm{a}^{2}}{L^{2}}\right]+\cdots
$$

This formula has been extended to more than two bosons in a box in $[9,10]$.

Generalization of to higher partial waves was also done in [4]. In this case complications arise due to breaking of rotational symmetry causing partial waves to mix. For s-wave scattering (angular momentum $l=0$ ) the lowest wave that mixes in is $l=4$ and it's effects can be ignored in most cases. In general, these mixing effects cannot be ignored, and the scattering phase shifts $\delta_{l}$ can be determined as the zeros of a characteristic polynomium defined by

$$
\operatorname{det}\left[e^{2 i \delta}-\mathbf{U}\right]=0
$$

with $\mathbf{U}$ and appropriately constructed matrix for each symmetry sector of the cubic group, and $\delta$ a diagonal matrix with entries the phase shifts of each partial wave contributing to the cubic symmetry sector at hand. In general, there are infinitely many partial waves contributing to each 

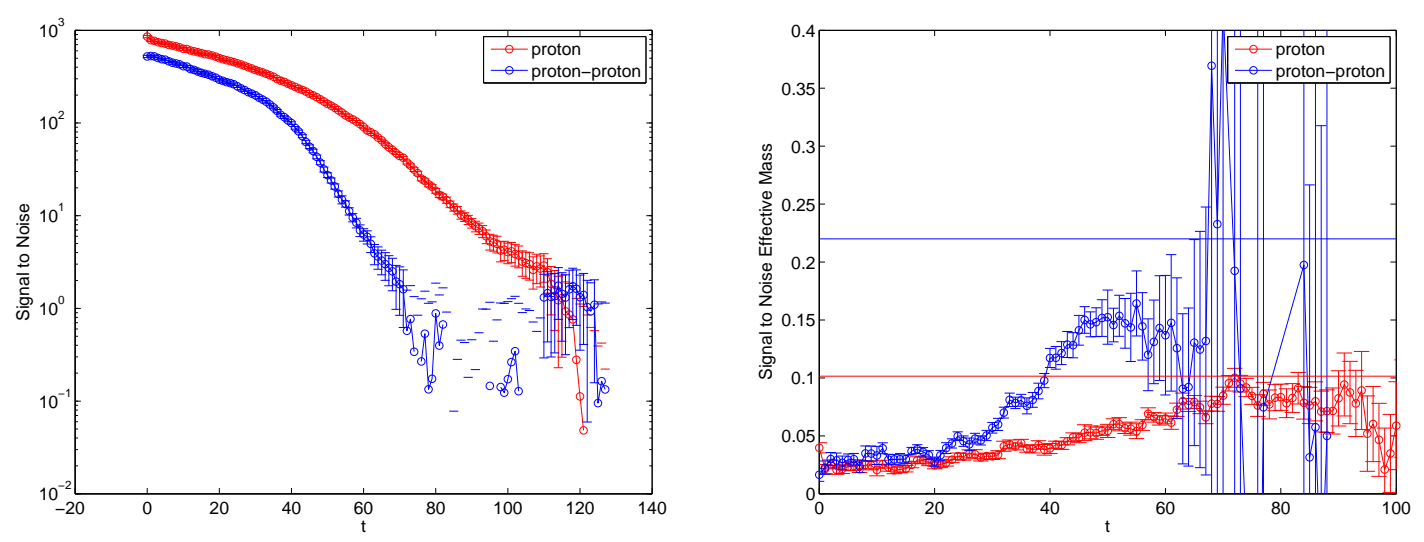

Figure 1: Signal to noise ratio for a single proton and a two proton system. Left, the signal to noise ratio. Right, the logarithmic derivative of the signal to noise ratio.

cubic symmetry sector, however, in practice the matrix is truncated to a small number of partial waves. For details of this formalism see [4] and the more recent [11].

Generalizations of the above formalism to non-center of mass frames can be found in $[12,13$, 14, 15]. In addition, the extension to non-cubic boxes can be found at [16]. Finally, extensions to multi-channel scattering have been worked out in [17].

When two particles interact attractively, the possibility of the formation of a bound state arises. In this case the energy splitting $\Delta E_{0}$ is negative resulting in an imaginary scattering momentum $k=i \kappa$,

$$
\Delta E_{0}=2 \sqrt{k^{2}+\mu^{2}}-2 \mu \approx \frac{k^{2}}{2 \mu}=-\frac{\kappa^{2}}{2 \mu} .
$$

It has been shown [18] that in the case of bound states the volume dependence of $\Delta E_{0}$ is exponential rather than the power law found for scattering states. Recently, in [19] the finite volume energy shift for angular momentum $l>0$ bound states was computed:

$$
\Delta E_{0} \sim \alpha\left(\frac{1}{\kappa L}\right) \frac{e^{-\kappa L}}{\mu L}+\mathscr{O}\left(e^{-\sqrt{2} \kappa L}\right) .
$$

where the function $\alpha(x)$ depends on the partial wave as well as the lattice symmetry sector this partial wave is embeded. A notable result of this work is the fact that while s-wave bound states become more deeply bound in finite volume, $\mathrm{p}$-wave bound states are less bound in small volume.

\section{Signal to Noise ratio for baryons}

Following the formalism described in the previous section the calculation of elastic scattering phase shifts from lattice QCD is reduced to the calculation of the energy spectrum of two bodies in a finite box. This is in principle straight forward to do. Methods for extracting not only the ground state but several low lying energy levels exist $[5,20]$. In the case of baryons, however, the extraction of the spectrum of the multi-body system in a box is quite difficult. Here the problem 
is two fold. First, the energy gaps in the two (or multi) baryon systems are small requiring large Euclidean time to project out the ground state (or a set of few low-lying states). The cost of quark contractions is such that it makes it very difficult to build a sufficiently large basis of operators (as is now done for single baryon spectroscopy [21, 22, 23]). Secondly, the statistical noise in the correlators due to Monte Carlo sampling grows exponentially with Euclidean time.

As Lepage showed [24], the signal to noise ratio $\mathscr{R}$ of hadronic correlation functions drops exponentially with Euclidean time with an exponent related to the gap between the mass of the pion and the mass of the the lowest hadronic state contributing to the correlator. In particular, for a system with $A$ nucleons we have,

$$
\mathscr{R} \approx e^{-A\left(m_{N}-\frac{3}{2} m_{\pi}\right) t}
$$

where $m_{N}$ is the nucleon mass and $m_{\pi}$ the pion mass and assuming weak interaction between nucleons. Fortunately, as it was shown in $[25,26]$ this asymptotic bound does not set in at early times leaving some region where reasonable accuracy can be achieved with sufficiently high statistics. In Fig. 1, I show the drop of the signal to noise ratio for the case of a single proton as well as the case of a two proton system in the singlet spin state. In both cases after a region of a mild drop the exponential rapid decay sets in. In the case of the proton, we can follow the signal at sufficiently large time to observe the saturation of the Lepage bound, as it is seen in the right panel of Fig. 1. For the two proton system the correlator becomes too noisy as the Lepage bound is approached, never quite saturating the bound. The data I present here are from NPLQCD collaboration for an ensemble with dynamical anisotropic clover fermions with a $390 \mathrm{MeV}$ pion mass with anisotropy of about 3.5 and volume of $32^{3} \times 256$ and a spacial lattice spacing of about $0.125 \mathrm{fm}$.

The issue of the rapid growth of the noise as well as the dense two body spectrum in a box present a major challenge that needs to be overcome, for reaching precision calculations of properties of systems with interacting baryons.

\section{Meson Scattering}

In the case of pions in maximal isospin states (such as the $\mathrm{I}=2, I_{z}=2$ channel), no exponential growth of the error in the correlators is observed, simply because the exponent in the Lepage formula vanishes. In addition, pions being very light, the two body (or multi-body) spectrum in a box has wider energy splittings. The combination of these two effects results in a very precise calculation of the $\mathrm{I}=2 \pi-\pi$ elastic scattering phase shift. Nonetheless, new improved methods have been developed, applied and tested on $\pi-\pi$ systems [31,30]. There have been numerous lattice calculations over the last few years of the $\mathrm{I}=2 \pi-\pi$ scattering length. In Fig. 2, I present a compilation of recent results of the $\mathrm{I}=2 \pi-\pi$ s-wave elastic scattering length. The results presented are extrapolated at the physical pion mass. The calculations by NPLQCD [32, 26] and ETMC [33] are from a direct lattice calculation. NPLQCD results are on a single lattice spacing while ETMC worked on two lattice spacings in an effort to control discretization errors. For comparison, in Fig. 2 I show the experimental results from NA47 [27, 28] and E865 [29] collaborations, a result obtained by Colangelo et al. [34] using experimental data and chiral symmetry constraints, as well as indirect lattice determinations using chiral Lagrangian low energy constants extracted from lattice calculations by MILC. It is remarkable that today, lattice calculations are providing the most precise determination of the pion s-wave elastic scattering length at the $\mathrm{I}=2$ channel. 


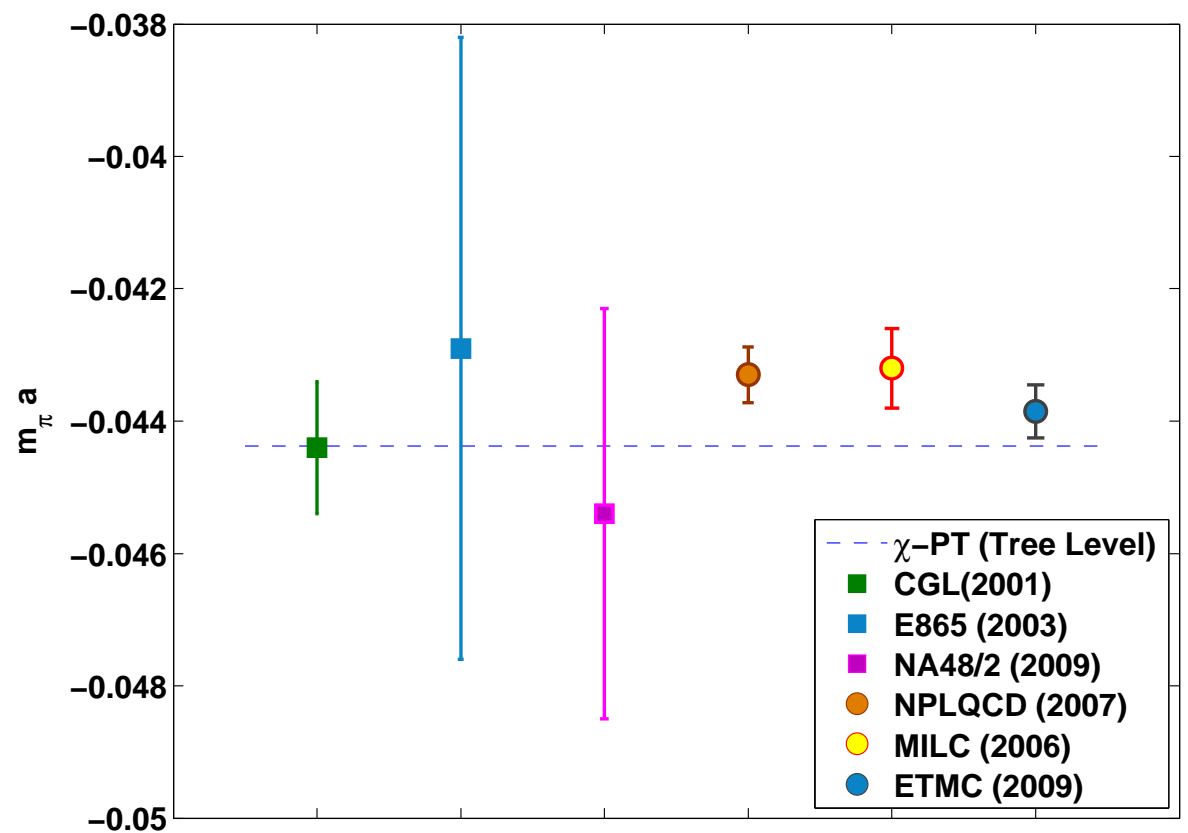

Figure 2: World lattice QCD data on $\pi-\pi \mathrm{I}=2$ elastic s-wave scattering length. Results from MILC collaboration are determined indirectly using chiral Lagrangian low energy constants extracted from lattice calculations. The experimental results denoted by the magenta and blue squares are from NA47 [27, 28] and E865 [29] collaborations respectively. For lattice results systematic and statistical errors are combined in quadrature.

As we discussed above, the full scattering elastic phase shift can be computed as well. Pioneering work in the quenched approximation was performed by CP-PACS [35]. Recently, Dudek et al. [30] have also computed the s-wave $\mathrm{I}=2 \pi-\pi$ scattering phase shift using dynamical clover improved fermions on anisotropic lattices and pion masses heavier than the physical point. Their results are presented in Fig. 3. In the same calculation, the d-wave scattering phase shift was also obtained.

Apart from pion scattering processes lattice calculations of $K-\pi$ and K-K scattering lengths have been performed recently. A status of these calculations can be found in a recent review [26]. Finally, the study of resonances requires the calculation of the scattering phase shift in the appropriate channel. Several lattice calculations in the last few years have been geared in this direction. In this conference such calculations where reviewed in an other plenary by Bulava and are summarized in [36].

\section{Baryon-Baryon Interactions}

Much like mesons, calculations of baryon-baryon scattering lengths have also started many years ago with the pioneering work of Fukugita et al. [37] in the quenched approximation. It took ten years for the first calculation with dynamical quarks to appear by NPLQCD collaboration [38]. The issues related to the noise and the spectrum of two baryon systems in a box outlined in Sec. 3 


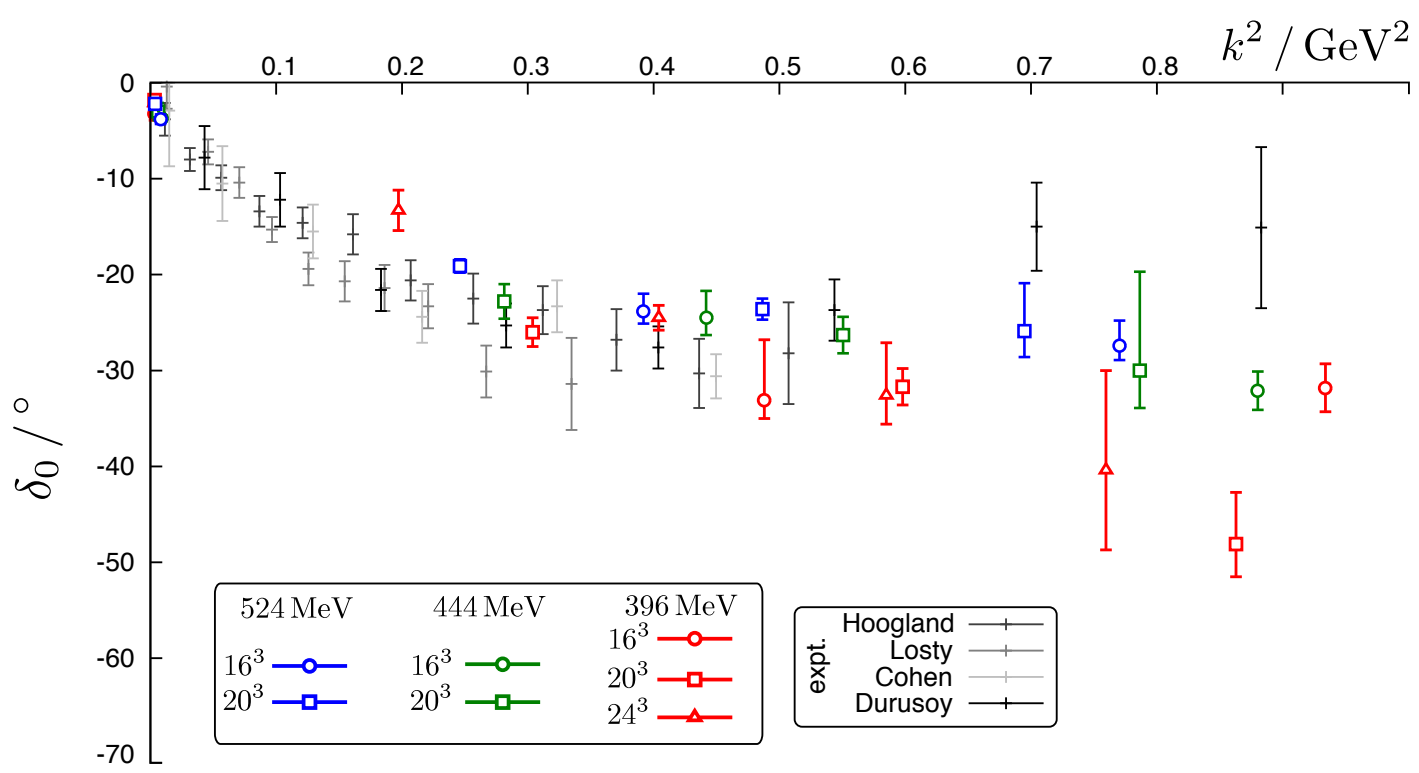

Figure 3: The s-wave scattering phase shift for several pion masses. Results are from [30].

are the prime reasons for the sparsity of results in the baryon sector in contrast to the meson sector. The state of nucleon-nucleon elastic scattering length calculations is summarized in Fig. 4 which taken from a recent review [26]. As one can see, there is a significant improvement in statistical errors in the recent results both from HALQCD [39] and and NPLQCD collaborations. However, systematic effects due to excited state contamination, heavy quark masses, and lattice spacing effects, are far from being well under control. In particular, no clear evidence of the bound deuteron in the triplet channel is observed in unquenched calculations up to the time of this talk, and the computed scattering lengths are far from being in agreement with the precisely measured experimental numbers. The fine tuned nature of nuclear physics close to the physical point might be one of the reasons of the clear disagreement between lattice and experimental results. On the other hand, these pioneering results represent the first solid step in the direction of obtaining reliable calculations of these important to Nuclear physics quantities. Clearly, in order to make progress improved methodology, as well as availability of significant resources is required.

In the last year a calculation of the binding energy of the deuteron was performed in the quenched approximation [40]. In the same calculation, a bound di-neutron was observed. The key features of these calculations were the big volumes (up to $12 \mathrm{fm}$ ), the heavy pion masses (800MeV), and the use of a variational method with 2 operators with wavefunctions designed to have large overlap with the ground state of the system. The combination of these choices makes the noise problem tractable. In Fig. 5 the results of this calculation are presented. In particular, the extrapolation to the infinite volume limit from several finite volume results are shown. In addition, results from the two operators used and two independent ensembles of gauge fields are presented. The status of these calculations were presented by Yamazaki in this conference. Furthermore, the same authors preformed similar calculations of the binding energy of the helium nuclei $\left({ }^{3} \mathrm{He}\right.$ and $\left.{ }^{4} \mathrm{He}\right)$ 

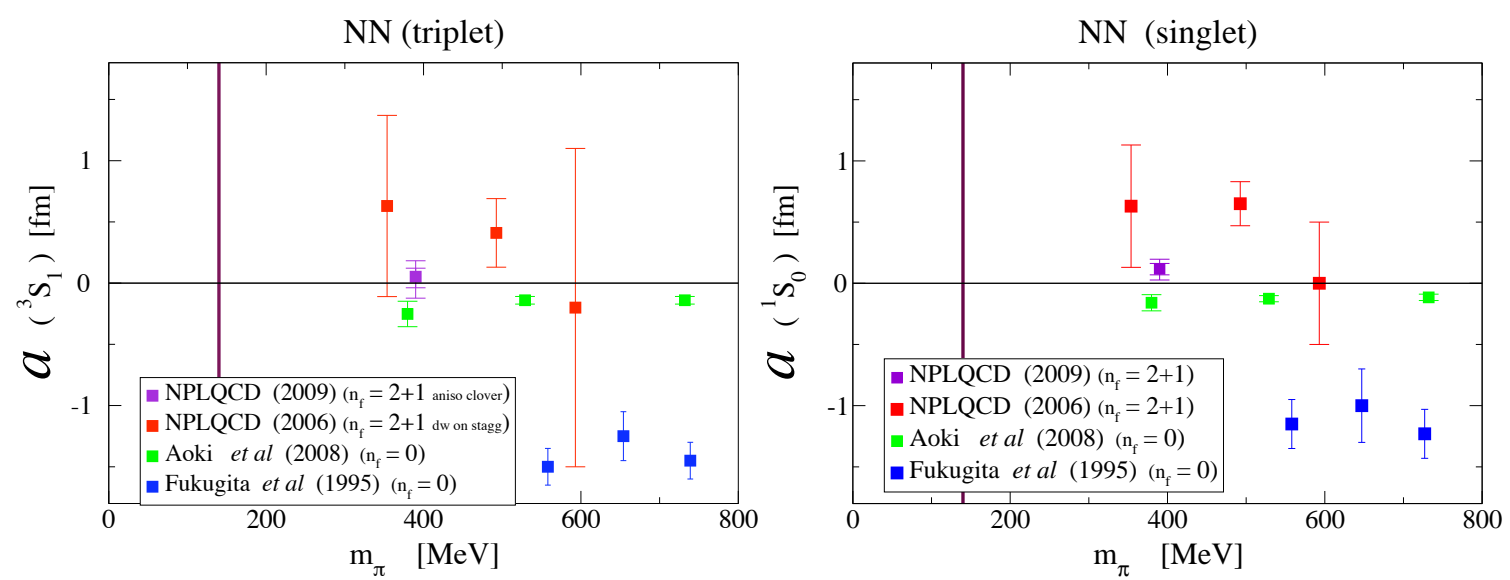

Figure 4: The elastic scattering length in the singlet and triplet spin channels of the two nucleon system.
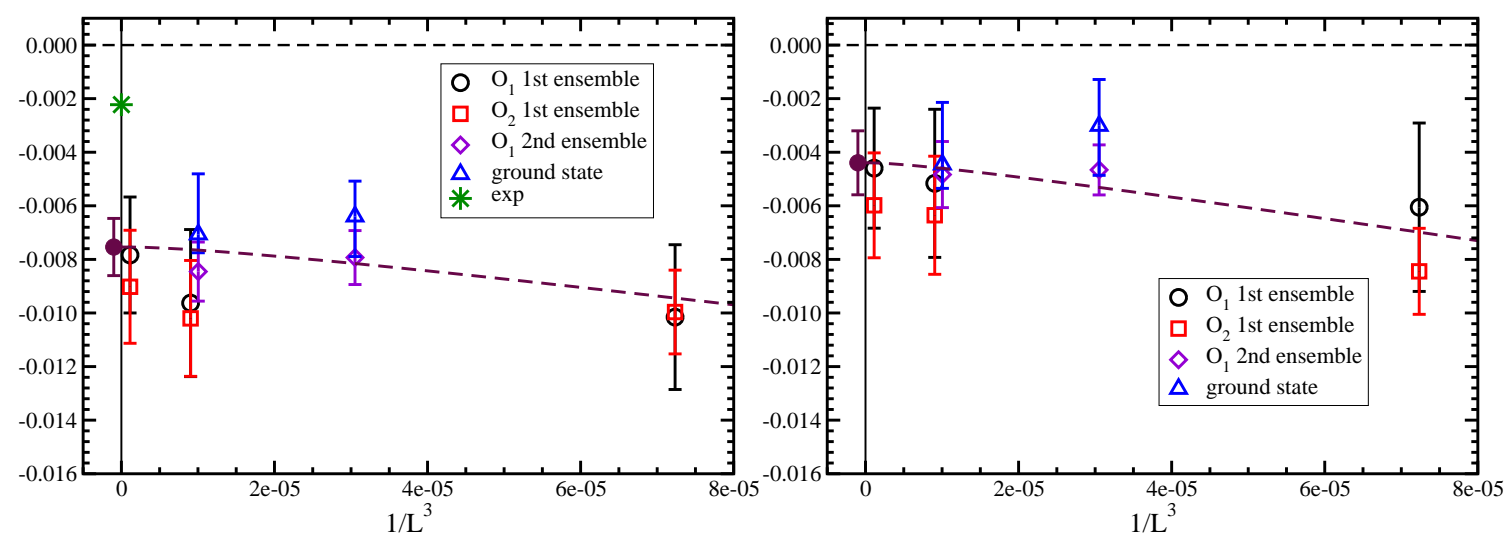

Figure 5: The binding energies of the deuteron (left) and di-neutron (right) computed in the quench approximation at a pion mass of approximately $800 \mathrm{MeV}$. Figures are from [40].

in quenched approximation [41].

\subsection{H-dibaryon}

In contrast to the status of elastic scattering phase shifts in the nucleon sector, significant progress occurred over the last year in exploring the existence of baryon number 2 bound states in QCD. Most notable was the work on the h-dibaryon by HALQCD and NPLQCD. The h-dibaryon as a possible six quark state (two of each of the three light flavors in the quark model picture) was proposed by Jaffe in 1977 [42]. This is a strangeness -2, baryon number 2, and angular momentum 0 state. Jaffe's argument was based on the MIT bag model and the perturbative attractive colorspin interactions that form di-quarks in his model. Since then there have been several experimental searches for the h-dibaryon. All this effort is comprehensively reviewed in [43]. Most recently, at RIHC [44] using some input from models, the region between 0 and $95 \mathrm{MeV}$ was excluded for the binding energy of the h-dibaryon. In addition, the possibility of a near threshold resonance is still not excluded by experimental results [45]. 
On the theory side there have been significant activity attempting to understand the existence of the h-dibaryon within various models. A review of all this work can be found in [46, 43]. Model predictions vary in a wide range, from deeply bound h-dibaryon to a resonance significantly above threshold. In lattice QCD calculations efforts to address the possibility of existence of the h-dibaryon started early on. In 1985 Mackenzie and Thacker [47] found evidence against the existence of a stable h-dibaryon in the quenched approximation. Subsequently, Pochinksy et al. [48] as well as Wetzorke et al. $[49,50]$ came to the same conclusion again in quenched approximation. Evidence to the contrary was reported by Iwasaki et al. in 1987 [51] again in quenched approximation. Similarly, the most recent quenched approximation calculation on anisotropic lattices with multiple volumes and lattice spacings [52], reported a bound h-dibaryon with a binding energy $B$

$$
B=70 \pm 11 \pm 15 \mathrm{MeV}
$$

where the first error is statistical and the second systematic.

During the last year the first unquenched calculations appeared. NPLQCD collaboration used anisotropic clover improved lattices with $2+1$ flavors at a single pion mass of $390 \mathrm{MeV}$ and a single spacial lattice spacing of approximately $0.125 \mathrm{fm}$. Details of these ensembles can be found in [53]. The calculation was performed on four volumes ranging between $2 \mathrm{fm}$ and $4 \mathrm{fm}$ and the infinite volume binding energy was calculated by extrapolation using the formalism outlined in Sec. 2 . This calculation provides evidence that at $390 \mathrm{MeV}$ pion mass the $\mathrm{h}$-dibaryon is bound with binding energy of

$$
B=13.2 \pm 1.8 \pm 4.0 \mathrm{MeV}
$$

where the first error is statistical and the second systematic. In addition to this number, NPLQCD has preliminary results at a lighter pion mass $(220 \mathrm{MeV})$. However the precision obtained at this mass is not sufficient to determine the sign of the binding energy. NPLQCD results as well as more details on their calculation can be found in $[54,55]$.

HALQCD also presented results on the h-dibaryon during the last year. Their work is on flavor $\mathrm{SU}(3)$ symmetric lattices and pion masses that range between $469 \mathrm{MeV}$ and $1.2 \mathrm{GeV}$. They also found evidence of a bound h-dibaryon with binding energies ranging from $26 \mathrm{MeV}$ up to $37 \mathrm{MeV}$. At the point close to the physical strange quark mass (pion mass of $837 \mathrm{MeV}$ ) their result is

$$
B=37.4 \pm 4.4 \pm 7.3 \mathrm{MeV}
$$

where the first error is statistical and the second systematic. There results were first reported in [56]. In this conference Inoue [57] reported a lighter SU(3) symmetric point at pion mass $469 \mathrm{MeV}$ with a binding energy of

$$
B=26.0 \pm 4.4 \pm 4.8 \mathrm{MeV}
$$

The approach used by the HALQCD collaboration is very different from this of NPLQCD. They rely on the extraction the Nambu-Bethe-Salpeter amplitude or "wave function" from lattice correlators with varying relative position of the two annihilation interpolating fields for the two baryons. Assuming energy independence as well as locality of the the Nambu-Bethe-Salpeter potential they extract the "potential" using the calculated "wave function". After the "potential" is obtained and parametrized on finite volume the infinite volume Schrödinger equation is solved 


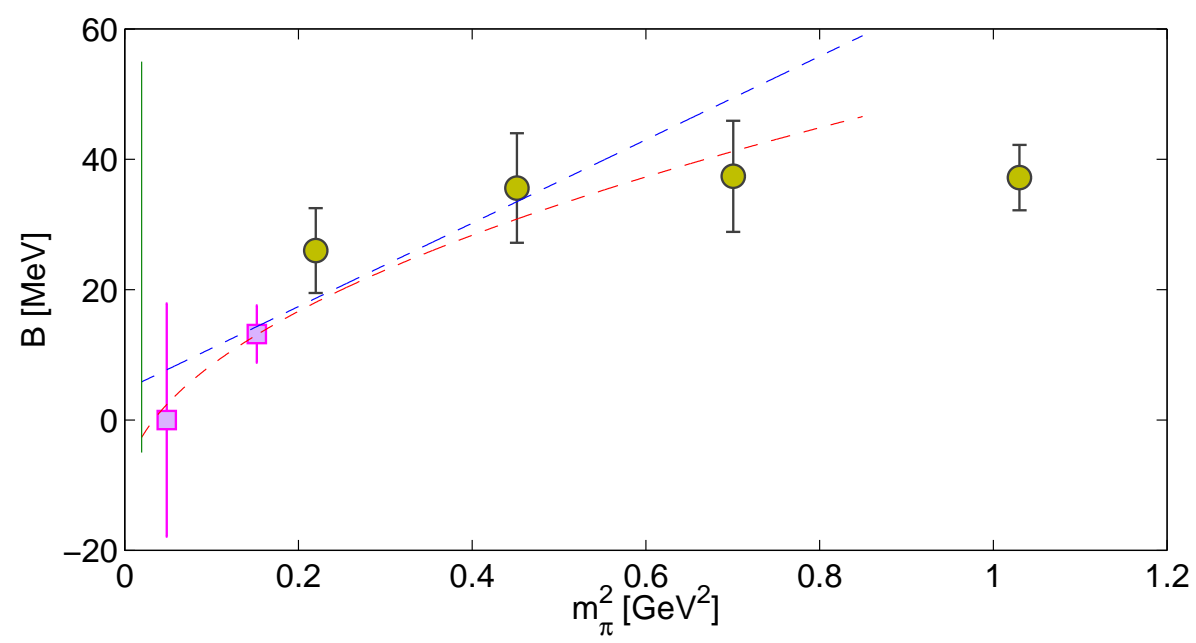

Figure 6: Summary of lattice results for the h-dibaryon binding energy. The squares are results from NPLQCD collaboration [54, 55] and the circles are results from HALQCD (in [56] and in this conference [57]). The solid vertical line marks the physical point. The two dashed lines are plotted to guide the eye (the blue is linear in $m_{\pi}^{2}$ while the red is linear in $m_{\pi}$ ).

to obtain the binding energy. Their methodology was presented in a Lattice 2010 plenary talk by Hatsuda [58] and hence I am not going to elaborate more here. Both assumptions made, however, are in principle not true as explained in [2,4]. In addition, the dependence of the final result to the choice of single nucleon interpolating fields also needs investigation. HALQCD has made a significant effort in understanding how these assumptions affect the calculated binding energies (and phase shifts in other cases) and their results are published in [59] and the recent review by Aoki [60].

The summary of the unquenched results up to this conference for the h-dibaryon binding energy are presented in Fig. 6. As it is evident, the binding energy is decreasing as the pion mass approaches the physical point. In addition, at lighter pion masses the calculations become harder providing less accurate results. For these reasons, although a bound h-dibaryon is observed at heavier pion masses, it is not clear what its fate is at the physical point. Apart from obtaining precision close to the physical point, lattice calculations will have to address and control other remaining systematic errors such as lattice spacing effects, isospin breaking and electromagnetic effects. Given the fact that the h-dibaryon may be a near threshold sate (bound or resonance) all these effects have to be carefully addressed before a final result is obtained from lattice QCD. It is encouraging though, that during the last year significant progress has been made. In addition to lattice calculations, in the literature recently appeared work that address the quark mass dependence of the binding energy of the h-dibaryon [61] using chiral symmetry constraints. In this case, the binding energy also seems to decrease with the pion mass.

\section{Other multi-hadron systems}

In this conference several other works studying the properties of multi hadron systems were 
presented. Ikeda for HALQCD reported on their efforts to extract information about meson-baryon interactions from lattice QCD [62]. NPLQCD had published work on meson baryon scattering lenghts few years ago in [63]. In addition, hyperon-nucleon interaction studies were reported in this conference by Nemura for HALQCD[64]. In the framework of mixed action calculations and more recently using anisotropic clover fermion ensembles NPLQCD performed calculations of hyperon-nucleon scattering lengths [25, 65] as well. Finally, Doi reported on the status of the three nucleon interaction calculations for HAL QCD [66] using the Nambu-Bethe-Salpeter wavefunction approach.

New results on the properties of multi-pion systems were reported by Shi [67]. In his calculation, new methods were employed to construct correlation functions up to 72 pions on multiple volumes allowing explorations of properties of matter at non-zero isospin density. This work represents a novel approach in the study of systems with non-zero isospin density that might shed new light to the problem.

\section{Conclusions}

There has been significant progress in the last few years in studies of multi-hadron systems in lattice QCD. The ultimate goal being understanding how nuclear physics emerges from QCD. The challenges in tackling this problem are quite difficult, however, the emergence of new methods, and the expected significant increase in computer cycles available to us, makes me optimistic that we will continue on this pace of steady progress in the years to come.

\section{Acknowledgments}

This work was supported in part by the DOE grants DE-AC05-06OR23177 (JSA) and DEFG02-04ER41302 and in part by the NSF grant CCF-0728915.

\section{References}

[1] M. Luscher (1983)

[2] M. Luscher, Commun.Math.Phys. 105, 153 (1986)

[3] L. Maiani, M. Testa, Phys.Lett. B245, 585 (1990)

[4] M. Luscher, Nucl.Phys. B354, 531 (1991)

[5] M. Luscher, U. Wolff, Nucl.Phys. B339, 222 (1990)

[6] M. Luscher, Nucl.Phys. B364, 237 (1991)

[7] L. Lellouch, M. Luscher, Commun.Math.Phys. 219, 31 (2001), hep-lat/0003023

[8] C. Lin, G. Martinelli, C.T. Sachrajda, M. Testa, Nucl.Phys. B619, 467 (2001), hep-lat/0104006

[9] S.R. Beane, W. Detmold, M.J. Savage, Phys.Rev. D76, 074507 (2007), 0707.1670

[10] S. Tan, Phys. Rev. A 78, 013636 (2008), http://link.aps.org/doi/10.1103/PhysRevA.78.013636

[11] T. Luu, M.J. Savage, Phys.Rev. D83, 114508 (2011), 1101.3347 
[12] S.A. Gottlieb, K. Rummukainen, Nucl.Phys.Proc.Suppl. 47, 819 (1996), hep-lat/9509088

[13] N.H. Christ, C. Kim, T. Yamazaki, Phys.Rev. D72, 114506 (2005), hep-lat/0507009

[14] C. Kim, C. Sachrajda, S.R. Sharpe, Nucl.Phys. B727, 218 (2005), hep-lat/0507006

[15] X. Feng, K. Jansen, D.B. Renner (ETM Collaboration), PoS LAT2010, 104 (2010), 1104.0058

[16] C. Miao, X.i. Du, G.w. Meng, C. Liu, Phys.Lett. B595, 400 (2004), hep-lat/0403028

[17] S. He, X. Feng, C. Liu, JHEP 0507, 011 (2005), hep-lat/0504019

[18] M. Luscher, Commun.Math.Phys. 104, 177 (1986)

[19] S. Koenig, D. Lee, H.W. Hammer, Phys.Rev.Lett. 107, 112001 (2011), 1103.4468

[20] B. Blossier, M. Della Morte, G. von Hippel, T. Mendes, R. Sommer, JHEP 0904, 094 (2009), 0902.1265

[21] R.G. Edwards, J.J. Dudek, D.G. Richards, S.J. Wallace, Phys.Rev. D84, 074508 (2011), 1104.5152

[22] C. Morningstar, A. Bell, J. Bulava, J. Foley, K. Juge et al., AIP Conf.Proc. 1388, 34 (2011), 1103.2783

[23] C. Morningstar, A. Bell, C. Chen, D. Lenkner, C. Wong et al., PoS ICHEP2010, 369 (2010), 1011.6573

[24] P.G. Lepage, TASI'89 ASI 1989 pp. 97-120 (1989)

[25] S.R. Beane et al. (NPLQCD Collaboration), Phys.Rev. D81, 054505 (2010), 0912.4243

[26] S. Beane, W. Detmold, K. Orginos, M. Savage, Prog.Part.Nucl.Phys. 66, 1 (2011), 1004.2935

[27] J. Batley, A. Culling, G. Kalmus, C. Lazzeroni, D. Munday et al., Eur.Phys.J. C64, 589 (2009), 0912.2165

[28] J.R. Batley et al. (NA48-2), Eur. Phys. J. C70, 635 (2010)

[29] S. Pislak, R. Appel, G. Atoyan, B. Bassalleck, D. Bergman et al., Phys.Rev. D67, 072004 (2003), hep-ex/0301040

[30] J.J. Dudek, R.G. Edwards, M.J. Peardon, D.G. Richards, C.E. Thomas, Phys.Rev. D83, 071504 (2011), 1011.6352

[31] J. Bulava et al. (For the Hadron Spectrum Collaboration), PoS LATTICE2010, 110 (2010), 1011.5277

[32] S.R. Beane, T.C. Luu, K. Orginos, A. Parreno, M.J. Savage et al., Phys.Rev. D77, 014505 (2008), 0706.3026

[33] X. Feng, K. Jansen, D.B. Renner, Phys.Lett. B684, 268 (2010), 0909.3255

[34] G. Colangelo, J. Gasser, H. Leutwyler, Nucl.Phys. B603, 125 (2001), hep-ph/0103088

[35] S. Aoki et al. (CP-PACS Collaboration), Phys.Rev. D67, 014502 (2003), hep-lat/0209124

[36] J. Bulava, PoS(Lattice 2011) (2011), 1112.0212

[37] M. Fukugita, Y. Kuramashi, M. Okawa, H. Mino, A. Ukawa, Phys.Rev. D52, 3003 (1995), hep-lat/9501024

[38] S. Beane, P. Bedaque, K. Orginos, M. Savage, Phys.Rev.Lett. 97, 012001 (2006), hep-lat/0602010

[39] S. Aoki, T. Hatsuda, N. Ishii, Comput.Sci.Dis. 1, 015009 (2008), 0805.2462 
[40] T. Yamazaki, Y. Kuramashi, A. Ukawa, Phys.Rev. D84, 054506 (2011), 1105.1418

[41] T. Yamazaki, Y. Kuramashi, A. Ukawa, f.t.P.C. Collaboration, Phys.Rev. D81, 111504 (2010), 0912.1383

[42] R.L. Jaffe, Phys. Rev. Lett. 38, 195 (1977)

[43] T. Sakai, K. Shimizu, K. Yazaki, Prog.Theor.Phys.Suppl. 137, 121 (2000), nucl-th/9912063

[44] A.L. Trattner, Ph.D. thesis, UMI-32-54109 (2006)

[45] C.J. Yoon et al., Phys. Rev. C75, 022201 (2007)

[46] S. Bashinsky, R. Jaffe, Nucl.Phys. A625, 167 (1997), hep-ph/9705407

[47] P.B. Mackenzie, H. Thacker, Phys.Rev.Lett. 55, 2539 (1985)

[48] A. Pochinsky, J.W. Negele, B. Scarlet, Nucl.Phys.Proc.Suppl. 73, 255 (1999), hep-lat/9809077

[49] I. Wetzorke, F. Karsch, E. Laermann, Nucl.Phys.Proc.Suppl. 83, 218 (2000), hep-lat/9909037

[50] I. Wetzorke, F. Karsch, Nucl.Phys.Proc.Suppl. 119, 278 (2003), hep-lat/0208029

[51] Y. Iwasaki, T. Yoshie, Y. Tsuboi, Phys.Rev.Lett. 60, 1371 (1988)

[52] Z.H. Luo, M. Loan, X.Q. Luo, Mod.Phys.Lett. A22, 591 (2007), 0803.3171

[53] H.W. Lin et al. (Hadron Spectrum Collaboration), Phys.Rev. D79, 034502 (2009), 0810.3588

[54] S. Beane et al. (NPLQCD Collaboration), Phys.Rev.Lett. 106, 162001 (2011), 1012.3812

[55] S. Beane, E. Chang, W. Detmold, B. Joo, H. Lin et al., Mod.Phys.Lett. A26, 2587 (2011), 1103.2821

[56] T. Inoue et al. (HAL QCD Collaboration), Phys.Rev.Lett. 106, 162002 (2011), 1012.5928

[57] T. Inoue (for HAL QCD Collaboration) (2011), 1111.5098

[58] T. Hatsuda, PoS(Lattice 2010) p. 011 (2010)

[59] K. Murano, N. Ishii, S. Aoki, T. Hatsuda, Prog.Theor.Phys. 125, 1225 (2011), 1103.0619

[60] S.A. for HAL QCD, Prog.Part.Nucl.Phys. 66, 687 (2011), 1107.1284

[61] P. Shanahan, A. Thomas, R. Young, Phys.Rev.Lett. 107, 092004 (2011), 1106.2851

[62] Y. Ikeda (for HAL QCD Collaboration) (2011), 1111.2663

[63] A. Torok, S.R. Beane, W. Detmold, T.C. Luu, K. Orginos et al., Phys.Rev. D81, 074506 (2010), 0907.1913

[64] H. Nemura (for HAL QCD Collaboration) (2012), 1203.3320

[65] S.R. Beane et al. (NPLQCD Collaboration), Nucl.Phys. A794, 62 (2007), hep-lat/0612026

[66] T. Doi (HAL QCD Collaboration) (2011), 1112.4103

[67] Z. Shi, W. Detmold, PoS LATTICE2011, 328 (2011), 1111.1656 